Gottdiener, Mark and Alexandros Ph. Lagopoulos (eds.) (1986): The City and the Sign. New York: Columbia Universtiy Press.

Ha'aretz editorial (2005): Citizenship for the Children. Ha'aretz English Edition, November 2.

Hammer, L. (1999): Migrant Workers in Israel: Towards Proposing a Framework of Enforceable Customary International Human Rights. Netherlands Quarterly of Human Rights 171: 5-30.

Kemp, Adriana and Rebeca Raijman (2004): Tel Aviv is not Foreign to you: Urban Incorporation Policy on Labor Migrants in Israel. International Migration Review 38 (1): 26-51.

Leibovich-Dar, Sara (2003): Cleaned Out. Ha'aretz Magazine, English Edition, September 19.

Levine, Mark (2005): Overthrowing Geography. Jaffa, Tel Aviv, and the Struggle for Palestine, 1880-1948. Berkeley, Los Angeles, London: University of California.

Low, Setha (Hg.) (1999): Theorizing the City. The New Urban Anthropology Reader. New Brunswick. Rutgers University Press.

Mezzadra, Sandro (2007): Kapitalismus, Migrationen, soziale Kämpfe. Vorbemerkungen zu einer Theorie der Autonomie der Migration. M. Pieper et al. (eds.): Empire und die biopolitische Wende. Die intemationale Diskussion im Anschluss an Hardt und Negri. Frankfurt, New York: Campus Verlag, 179-193.

Mitchell, Don (2003): The Right to the City. Social Justice and the Fight for Public Space. New York: Guilford Press.

Neah, Boki and Dudi Nessim (2004): Police Without Mercy. Yedioth Ahronot, February 16. [Hebräisch]

Neah, Boki, Nessim, Dudi, Paz, Shali and Ilan Spira (2004): Deportation on Shabath. Yediot Ahronot, May 23. [Hebräisch]

Parreñas, Rhazel Salazar (2001): Servants of Globalization. Stanford: University Press.

Reily, Miri (2003): So long Allenby. Ha'aretz Magazine, English Edition, October 7.

Ribas-Mateos, Natalia (2005). The Mediterranean in the Age of Globalization: Migration, Welfare, And Borders. New Brunswick: Transaction Publisher.

Rosenhek, Zeev (1999): The Politics of Claims-Making by Labour Migrants in Israel. Journal of Ethnic and Migration Studies 25(4): 575-595.

Rosenhek, Zeev (2000): Migration Regimes, Intra-State Conflicts and the Politics of Exclusion and Inclusion: Migrant Workers in the Israeli Welfare State. Social Problems 47(1): 49-67.

Rotbard, Sharon (2005): White City, Black City. Tel Aviv: Babel. [Hebräisch]

Sassen, Saskia (1996): Analytic Borderlands: Race, Gender and Representation in the New City. S. 183-202, in: King, Anthony D., (Hg.): Re-Presenting the City: Ethnicity, Capital and Culture in the Twenty-First Century Metropolis. London. Macmillan Press.

Sassen, Saskia (2000): Cities in a World Economy. Boston: Pine Forge Press.

Schmitz, Thorsten (2007): Vermischtes aus Tel Aviv. Süddeutsche Zeitung, 4./5. August

Schnell, Itzhak (1999): Foreign Workers in Southern Tel Aviv - Yafo. Jerusalem: Floersheimer Institute for Policy Studies.

Semyonov, Moshe and Noah Lewin-Epstein (1987): Hewers of Wood and Drawers of Water Non-citizen Arabs in the Israeli Labour Market. Ithaca, NY: ILR Press.

Short, John Rennie, Breitbach, Carrie, Buckman, Steve and Jamey Essex (2000): From world cities to gateway cities: Extending the boundaries of globalization theory. City 4(3): 317-340

Transit Migration Forschungsgruppe (Hg.) (2007): Turbulente Ränder. Neue Perspektiven auf Migration an den Grenzen Europas. Bielefeld: transcript

Werbner, Pnina (1999): Global Pathways. Working Class Cosmopolitans and the Creation of Transnational Ethnic Worlds. Social Anthropology 7(1): 17-35.

Wurgaft, Nurit (2003): The Tenure of the Foreign Workers' Leaders is Very Short. Afterwards Comes Deportation. Ha'aretz, May 6. [Hebräisch]

Yeoh, Brenda S. A. and Shirlena Huang (1998): Negotiating Public Space: Strategies and Styles of

Migrant Female Domestic Workers in Singapore. Urban Studies 35(3): 583-602.
Matthias Bernt, Malte Daniljuk, Andrej Holm

\section{Informelle Urbanisierung, Selbstorganisation und "Sozialismus des 21. Jahrhunderts"}

Partizipative Stadtentwicklung in den Barrios von Caracas

Informelle Urbanisierungsprozesse sind in den Metropolen des Südens ein zentrales Merkmal der Stadtentwicklung. Städte wie Lagos, Nairobi, Mumbai oder Caracas bestehen zu einem Großteil aus Slums, die durch mangelhafte Versorgung mit Basisdienstleistungen wie Wasser und Strom, Substandardwohnen, Überbelegung, ungesunde Lebensbedingungen und ungeklärte Besitzverhältnisse gekennzeichnet sind. Weltweit betreffen solche Lebensumstände nach Schätzung von UN-Habitat 837 Mio. Menschen (UN-Habitat 2003: 13). Die globale Slumbewohnerschaft umfasst damit fast ein Achtel der gesamten Erdbevölkerung, wobei ihr Anteil im letzten Jahrzehnt, vor allem als Folge der neoliberalen Strukturanpassungsmaßnahmen der 1990er, noch rapide anwuchs. Urbanisierung heißt in den meisten Entwicklungsländern daher vor allem Ausbreitung von Slums - global gesehen bewegen wir uns in Richtung eines „Planeten der Slums“" (Davis 2007).

Eine zentrale Stellung unter den vielen verschiedene Formen von Slums (vgl. UN-Habitat 2003: 80ff.) nehmen squatter settlements ein, in Selbsthilfe ohne Genehmigung des Eigentümers und in Widerspruch zu planungs- und baurechtlichen Bestimmungen erbaute städtische Wohnviertel. Obwohl „illegal“, umfassen diese Shantytowns, Barrios, Bidonvilles, Gecekondus und Favelas heutzutage oft beträchtliche Größenordnungen und stellen in verschiedenen Städten bereits den Großsteil des gesamten Wohnungsangebotes dar.

Die damit einhergehenden „informellen Urbanisierungsprozesse“ sind weder neu (vgl. Neuwirth 2006, Davis 2007) noch kann man sagen, dass sie bislang keine politische Aufmerksamkeit erfahren hätten. Der Umgang mit illegal errichteten Siedlungen steht im Gegenteil bereits seit den 1960ern regelmäßig auf der entwicklungspolitischen Agenda vieler internationaler Organisationen. Weltbank und Vereinte Nationen haben seit Jahrzehnten Programme für squatter settlements im Angebot.

Allerdings hat sich dabei auch der Fokus internationaler Slumpolitik erheblich verändert: Kann die dominante Haltung gegenüber illegal errichteten Siedlungen noch in den 80er Jahren als Mischung aus Ignoranz und Ablehnung beschrieben werden, gelten heute Enabling, Selbsthilfe und Partizipation als Best 
Practice (UN-Habitat 2003: 132). In der Anwendung dieser Rezepte tut sich jedoch ein interessanter Widerspruch auf. Denn obwohl participatory slum improvement heute nahezu flächendeckend als Rezept zur Lösung der Wohnungskrise verkauft und mit erheblichen nationalen und internationalen Fördermitteln unterstützt wird, wächst nicht nur die Slumbevölkerung weiterhin rasant an, sondern die oft in der Literatur besprochenen „beispielhaften“ Projekte konnten insgesamt bisher nur „on a limited scale or (as) demonstration projects"(ebd.) realisiert werden. Zwischen Anspruch und Wirklichkeit klafft also eine erhebliche Lücke. Der Grund für die begrenzte Wirksamkeit internationaler Projekte liegt dabei weniger in einem Mangel an Konzepten oder zur Verfügung stehenden Ressourcen, sondern eher in den globalen und lokalen Machtverhältnissen, die dazu tendieren, die Benachteiligung von Slumbewohnern zu verstärken und eine Aufwertung und Integration der Wohnorte diskriminierter Bevölkerungsteile zu verhindern. Probleme des Umgangs mit „informellen Siedlungen" gehen deshalb weit über bauliche, infrastrukturelle und planerische Probleme hinaus und betreffen im Kern Fragen von sozialer Ungleichheit und Macht. Strategien für den Umgang mit der weltweit an Bedeutung gewinnenden informellen Urbanisierung dürfen daher nicht bei technischen Lösungen stehen bleiben, sondern müssen auch die politische Seite des Slumwohnens adressieren.

Vor diesem Hintergrund stellt die venezolanische Hauptstadt Caracas derzeit eines der interessantesten Experimente im Umgang mit Besetzersiedlungen dar. Denn zum Einen ist Caracas tatsächlich eine „Stadt der Barrios" und damit ein nahezu paradigmatisches Beispiel für informelle Urbanisierungsprozesse: Der Löwenanteil des Wohnungsbestandes wurde hier ohne Genehmigung errichtet und in Selbsthilfe erbaute Squattersiedlungen umschließen von überall sichtbar die in einem Talkessel liegende Innenstadt und die wohlhabenderen Wohnviertel. Zum Anderen finden derzeit in Caracas Veränderungen im Umgang mit diesen Siedlungen statt, die weltweit einmalig sind. Im Kontext eines „bolivarianischen“ sozialistischen Projektes (vgl. P.I.S.O. 16 2004, Twickel 2006, Azzelini 2006, Holm 2007) wird dabei die bis dahin marginalisierte Barriobevölkerung zum erstrangigen Adressaten nationaler Reformen gemacht. Diese beschränken sich nicht auf Sozialprogramme, sondern betreffen in zunehmendem Maße auch die Entscheidungsstrukturen der Stadtentwicklung. Das bestehende repräsentativ-hierarchische System der Stadtentwicklungsplanung wird dabei sukzessive um basisdemokratische Elemente erweitert, die die überkommenen Strukturen ersetzen, ergänzen und verändern. Die Tragweite der aktuellen Veränderungen ist immens, denn sie zielen nicht nur auf ein "Mehr" an Mitbestimmung und auf die Überwindung der die Metropole Caracas prägenden Benachteiligung der Barrios, sondern sie streben ein völlig neues System der kommunalen Politik an.

Ziel dieses Artikels ist es, diese venezolanische Erfahrung für ein deutsches Publikum zugänglich zu machen. Dabei geht es uns zum Einen darum, einen
Einblick in die Realitäten der viel diskutierten bolivarianischen „Revolution des 21. Jahrhunderts" „on the ground" zu geben und zu diskutieren, welche realen Veränderungen diese Umwälzungen für bis dato marginalisierte Bevölkerungsgruppen bereit halten. Zum Zweiten geht es uns darum, über die Auseinandersetzung mit den venezolanischen Reformen die in der internationalen Slumdiskussion oft an den Rand gedrängte politische Seite des Umgangs mit informellen Urbanisierungsprozessen stärker in den Vordergrund zu rücken.

Wir beschreiben hierfür zunächst Grundprobleme der Stadtentwicklung von Caracas, um so die Relevanz informeller Urbanisierungsprozesse in der venezolanischen Hauptstadt deutlich zu machen. Im Anschluss skizzieren wir Grundlinien der bolivarianischen Barriopolitik und erläutern anhand von zwei Beispielen im Detail, wie die aktuellen Neuerungen vor Ort aussehen.

\section{Caracas - Stadt der Barrios}

Die Stadtentwicklung von Caracas ist ein direktes Resultat des auf Ölexport basierenden venezolanischen Wirtschaftsmodells. Denn der Ölreichtum Venezuelas ermöglicht eine enorme Ölrente, die von den 1950er bis in die 1980er Jahren zu einer fordistisch-klientelistischen Variante nachholender Modernisicrung führte und ein immenses Wachstum sowie eine rasante Modernisierung der Stadt bezahlbar machte. Im Zuge einer enormen Expansion staatlicher Ausgaben entstanden im Zentrum der Stadt ein modernes Verkehrssystem, eine bemerkenswerte Infrastruktur und eine Vielzahl von ultramodernistischen Bauten. Die fordistische Modernisierung von Caracas erfasste allerdings nur das Zentrum der in einem Hochtal gelegenen Stadt. Parallel zu dieser Entwicklung des Zentrums breitete sich Caracas ungesteuert und explosiv in alle Richtungen aus. Die mit dem Bauboom und der Neuausrichtung der Volkswirtschaft auf eine rohstoffbasierte Rentenökonomie verbundene Nachfrage nach billigen und gering qualifizierten Arbeitskräften sowie die sich verschlechternde Situation auf dem Land führten zu einer gewaltige Zuwanderung in die Metropole. Da für die Zuwanderermassen trotz öffentlicher Wohnungsbauprogramme nie auch nur annähernd ausreichend Wohnraum geschaffen werden konnte, entstanden bereits in den 1940er Jahren die ersten Barrios. Diese dehnten sich schnell aus und bedeckten in den folgenden Jahrzehnten fast alle bis dahin unbebauten Flächen.

Da sowohl die Zuwanderung als auch die Bebauung weitgehend unkontrolliert erfolgten, wuchs die Bevölkerung der venezolanischen Hauptstadt so schnell, dass heute niemand genau weiß, wie viele Einwohner die Stadt hat. Sicher ist nur, dass Caracas sich innerhalb weniger Jahrzehnte von einer Kleinstadt zu einer Millionenmetropole entwickelte. Vallmitjana (1991) macht für das Wachstum der Stadt folgende Angaben: 1920 wohnten etwa 90.000 Einwohner in der Stadt Caracas, 1950 waren es schon fast 700.000, 1980 fast 3 Milli- 
onen. Die aktuelle Einwohnerzahl des Ballungsraums wird zwischen 3,2 Mio. und über 9 Mio. geschätzt.

Vor allem die später erbauten Barrios besiedelten dabei zunehmend die das Hochtal umgebenden Berghänge, und besetzten im Laufe der Jahre auch erosionsgefährdete Abhänge und überschwemmungsgefährdete Flussufer. Die Folge ist eine erhöhte Gefährdung der Bewohner durch Naturkatastrophen wie der vom Dezember 1999, als starke Regenfalle zum Abrutschen ganzer Viertel und zum Tod von etwa 30.000 Menschen durch Lawinen führten.

In Folge dieser städtebaulichen Geschichte ist Caracas, für jeden Besucher sofort erkennbar, eine zweigeteilte Stadt. Das Ziegelsteinrot der Barrios, die sich in Zwischenräumen unmittelbar an das Zentrum anschließen und bis in abenteuerliche Höhen an den Berghängen hochziehen, ist dabei die dominierende Farbe der Stadt. Entsprechend lebt die Mehrheit der Bevölkerung nicht im modernen Stadtzentrum, sondern in "informellen", im Selbstbau errichteten Siedlungen. „Informalität" ist damit das bestimmende Merkmal der Stadtentwicklung und es wird geschätzt, dass heute 40 (Marcano 2000) bis 60 Prozent (Grava et al. 2001) des gesamten Wohnungsbestandes aus illegal errichteten Siedlungen bestehen.

Die Entwicklung der Stadt erfolgte damit gemessen an europäischen Maßstäben „verkehrt herum": Sie kann als „inverse Urbanisierung" (Feireiss et al. 2005) verstanden werden und folgt der Formel Besetzung - Konstruktion Infrastruktur - Grundbucheintrag. Während in klassischen europäischen Städten Besitzverhältnisse lange geklärt sind, bevor Straßen und Häuser gebaut werden und die ersten Bewohner einziehen, ist es in den Barrios von Caracas in der Regel genau andersherum. In der Folge fehlen in weiten Teilen der Stadt nicht nur typisch städtische Einrichtungen, wie Straßen, Friedhöfe oder Wasserleitungen, sondern auch die Besitzverhältnisse waren oft nicht geklärt und Grundstücksgrenzen oder Baustandards erfahren keinerlei Beachtung.

Daraus resultiert eine ganze Reihe von Problemen, die hier nur stichwortartig skizziert werden können:

- Die Wohngebiete sind oft nur über improvisierte Treppen erschlossen.

- Die Straßenbeleuchtung fehlt in den meisten Fällen.

- Meist verfügen die Stadtteile nur über eine mangelhafte, mitunter auch über gar keine, Anschlüsse an Trinkwassernetze, Kanalisation, Stromversorgung oder Telefon.

- Wasser- und Stromversorgung werden durch „Anzapfen“ vorhandener Leitungen organisiert, was zu Ausfallzeiten, geringem Wasserdruck und Kurzschlüssen führt. Die Abwasserableitung über Sammelkanäle führt zu hygienischen Problemen und verstärkt zusätzlich die Bodenerosion.

Gerade in jüngeren und ärmeren Barrios existieren oft keine befestigten Straßen, so dass die Erschließung durch die in Caracas meist als Verkehrsmittel benutzten Minibusse kompliziert ist.
- Aufgrund der schlechten Erschließung ist die Müllentsorgung oft problematisch, weshalb an vielen Stellen „wilde“ Deponien zu finden sind; hieraus resultieren Probleme mit Ungeziefer, Emissionen und Hygiene.

- Die Ausstattung mit sozialer Infrastruktur, wie Schulen, Ärzten, Bibliotheken, Spielplätzen ist mangelhaft.

$\mathrm{Zu}$ diesen physischen Mängeln kommen eine Vielzahl von legalen Benachteiligungen und eine kulturelle Stigmatisierung der Barrios durch die kulturell die venezolanische Öffentlichkeit prägenden Mittelschichten, welche die Barrios als No-go-areas, als Horte der Gewalt und des Verbrechens stigmatisieren. Aus Sicht der Polizei waren die Barrios bis vor wenigen Jahren enemy territory (Perdomo \& Bolívar 1998: 124) und es gibt eine Vielzahl von Beispielen für die kriminalisierende und diskriminierende Behandlung ihrer Bewohner. Diese Stigmatisierung schlägt sich auch in einer legalen Benachteiligung nieder: Das Eigentum auf die in Selbsthilfe errichteten Häuser konnte so über Jahrzehnte hinweg nicht formal legalisiert werden, der Verstoß gegen Baunormen verunmöglicht den Abschluss legaler Mietverhältnisse und das Nichtvorhandensein städtischer Infrastrukturen macht in Zusammenwirken mit Verstößen gegen die offizielle Flächennutzungsplanung sogar den Abriss ganzer Stadtteile möglich.

Zusammenfassend kann man also sagen, dass das Leben im Barrio durch eine ganze Reihe von manifesten Problemen gekennzeichnet ist. Obwohl die Barrios Caracas mittlerweile dominieren, bedeutet, im Barrio zu wohnen, deshalb nach wie vor benachteiligt und von einer Vielzahl städtischer Versorgungsleistungen (Wasser, Strom, Müll, Verkehrswege) ausgeschlossen, rechtlich benachteiligt und kulturell stigmatisiert zu sein. Fasst man diese umfassende, soziale, kulturelle und legale Aspekte vereinigende Marginalisierung der Barrios zusammen, kann die Stadt Caracas als eine Art „Zwei-Klassen-Stadt“ verstanden werden. Das moderne Ideal einer „republikanischen“, egalitären, geplanten und rationalen Stadt gilt dabei in Gänze nur für das Zentrum und die Gebiete der wohlhabenden Schichten, während die Barrios und damit die Mehrzahl der Bewohner von Caracas jahrzehntelang von der Teilhabe ausgeschlossen wurden. In der Realität entwickelte sich damit nicht nur eine jedem Besucher anhand von Straßeneindrücken sofort deutliche kulturelle und soziale Spaltung der Stadt, sondern auch eine zunehmende disparate Auseinanderentwicklung von De-Facto-Normen und politischer Repräsentation. Pérez Perdomo und Bolívar sprechen in dieser Hinsicht von einem „legalen Pluralismus“, der Caracas kennzeichne und in dem formale Gesetze durch ,the law of the barrio" ergänzt würden.

\section{Von der Ignorierung über die Anerkennungen bis ...}

Dieses Auseinanderdriften von städtischen Realitäten und politischen Programmen wurde in der venezolanischen Stadtentwicklung lange Zeit einfach 
ignoriert. Obwohl seit den 1980er Jahren die Mehrzahl der Caraceños in illegal errichteten Siedlungen lebte, wurde die Benachteiligung dieser Bewohner lange Zeit überhaupt nicht thematisiert. Barrios galten, obwohl sie die Mehrheit der Stadt ausmachen, als „Marginalsiedlungen“, als „informel1" oder als "Ranchos" (in etwa „Hütten") und wurden vor allem als Problem behandelt. Dass die „ungeplante" Entwicklung von Caracas auch eine enorme Leistung der Selbstorganisation war, mit der städtische Probleme gelöst wurden, für die Staat und Markt keine Antwort hatten, wurde völlig negiert.

Allen Realitäten zum Trotz wurde die Verbreitung informeller Siedlungen von Staatsapparat, Fachleuten und öffentlicher Meinung über lange Zeit einfach abgelehnt. Bis weit in die 60er Jahre bestand die einzige Politikform gegenüber den Barrios in der Räumung, wobei niedergerissene Siedlungen in der Regel innerhalb kurzer Zeit an anderer Stelle wieder aufgebaut wurden. Ignoranz war die bestimmende Haltung: die informell entstandenen Stadtteile, die mittlerweile Hunderttausende BewohnerInnen zählten, blieben politisch und administrativ unsichtbar. Sie wurden nicht in die städtische Infrastrukturplanung einbezogen, erhielten keine politische Vertretung und tauchten nicht einmal im Stadtplan auf

Nur langsam, und auch weil mit zunehmendem Wachstum der Barrio-Stadt die Wählerstimmen aus den Barrios bedeutsamer wurden, entwickelten sich in den 80er Jahren zaghafte Versuche, die Barrios als Realität anzuerkennen und nicht nur Abrisse sondern auch Sanierungen, Infrastrukturmaßnahmen und Wohnumfeldverbesserungen durchzuführen. Der Rahmen für diese Ansätze war dabei vor allem klientelistischer Natur: Infrastrukturleistungen wurden als Wahlgeschenke verteilt und eine „neutrale“ Haltung der Behörden gegenüber Neubesetzungen bedurfte guter Kontakte zu lokalen Autoritäten. Fortschritte in den Barrios hingen dadurch zunehmend von der „Loyalität ${ }^{\prime \prime}$ des jeweiligen Viertels gegenüber gerade Ton angebenden Politikern und Beamten ab. Die wachsende Bedeutung der Barriobevölkerung für die venezolanischen Klientelsysteme eröffnete damit zwar Möglichkeiten für eine Verbesserung der Wohnverhältnisse und unterstützte auch die weitere Expansion der Barriostadt. Sie beschränkte aber gleichzeitig eine formale Integration der Barrios, in der eine Verbesserung der Wohnverhältnisse nicht mehr als "Geschenk“, sondern als „Recht" verankert gewesen wäre.

Legal anerkannt wurden dic Barrios erst 1987 mit einem neuen Stadtplanungsgesetz. Dieser Umschwung und die damit einhergehende Wende ist auf verschiedene Umstände zurückzuführen.

In Folge der Ölkrise 1982 kam es in Venezuela zu drastischen Ausgabenkürzungen und Sparmaßnahmen auf Kosten der Unterschichten. Hierdurch wurde nicht nur der zur Verteilung stehende Kuchen, und damit die Anreize für die Teilnahme an klientelistischen Strukturen verringert, sondern sukzessive erodierte auch die Legitimität der regierenden politischen Parteien. Die
Folge war eine sinkende Relevanz der klientelistischer Verteilungsstrukturen und ein Aufstieg ,informeller Entrepreneure ${ }^{6}$, die neue Landbesetzungen kommerziell organisierten und in bestehenden Barrios informelle Miet- und Bodenmärkte in Gang brachten (Perdomo/Bolivar 1998: 126).

Hinzu kommt, dass die Beschäftigung mit der Barrio-Stadt in der Fachöffentlichkeit der venezolanischen Stadtplaner und Architekten seit den 80er Jahren einen wachsenden Raum einnahm. Unter dem Slogan „Das Unsichtbare sichtbar machen" wurden in den 80er Jahren an der Universidad Central de Venezuela (UCV) verschiedene Studien zu den Wohn- und Lebensbedingungen in den Barrios durchgeführt, die eine andere Wahrnehmung dieser Viertel eröffneten (vgl. Bolívar et al. 1994, FVP 1991, Vallmitjana 1991).

Neben all diesen begünstigenden Faktoren ist aber vor allem das Entstehen und die Konsolidierung von sozialen Bewegungen von entscheidender Bedeutung, die in den $80 \mathrm{er}$ und 90er Jahren die Barriofrage zunehmend zum Politikum machten. Mit der Konsolidierung der Barrios als Nachbarschaften und einer Zuwendung linker Parteien zur Basisarbeit in den Armenvierteln kam es zur Herausbildung und Entwicklung von neuen sozialen Bewegungen, die aus den Barrios heraus agierten und die Situation in den Barrios zum Mittelpunkt ihrer politischen Alktionen machten. Oftmals spontan oder zur Lösung eines konkreten Problems gegründet, übernahmen Barriogruppen dabei Stück für Stück Verantwortung für einzelne Bereiche der Stadtteilorganisation. Von Kindergärten über Sportplätze bis hin zu Nachbarschaftszeitungen wurden etliche Aktivitäten damit nicht mehr vom Staat, sondern von den Nachbarschaftsorganisationen gewährleistet. Neben der eigenständigen Organisierung kam es dabei mit der Zeit auch zu einer zunehmenden Politisierung der Barriobewegungen. Konzentrierten sich Organisationsansätze und Aktivitäten in den Armenvierteln in den 70er und 80er Jahren oft vor allem auf kulturelle Projekte, rückten mit der Zeit auch Themen wie Wasserversorgung, der Zustand der Straßen und die juristische Absicherung der Siedlungen in den Vordergrund. Die bestehende Tradition von - oft eng mit den regierenden Parteien verflochtenen und in deren Klientelsysteme eingebundenen - Nachbarschaftsvereinigungen erfuhr damit einen entscheidenden Umbruch. Die Verarmung weiter Teile der Bevölkerung in Folge der neoliberalen Austeritätspolitik sowie die nach dem Caracazo-Aufstand und diversen Korruptionsaffären auf dem Nullpunkt angelangte Legitimation staatlicher Institutionen führten zu einem Aufschwung autonomer Initiativen, die in den $90 \mathrm{er}$ Jahren eine eigenständige politische und kulturelle Identität erlangten und sich zusehends ausweiteten und festigen konnten. Dabei kam es nicht nur zu einem politischen Bedeutungszuwachs der Nachbarschaftsvereine, sondern auch zu einer zunehmenden Vernetzung, Konsolidierung und eigenständigen Organisierung über das Lokale hinaus, die den Grundstein für viele der heutigen Aktivitäten legte. 


\section{Barriopolitik auf bolivarianisch}

Mit der Wahl 1998 und den politischen Veränderungen in Venezuela verbanden viele Aktivistinnen und Aktivisten aus den Barrios die Hoffnung auf eine neue Stadtentwicklungspolitik. Dass die Regierung Chávez, anders als ihre Vorgängerinnen, hier tatsächlich neue Schritte einleitete, versteht sich trotzdem nicht von selbst. Die darauf aufbauenden politischen Initiativen der Regierung folgten vielmehr - bei jeweils bedeutenden Unterschieden im Detail einer Reihe von Gemeinsamkeiten, die ihnen ihre typische Prägung geben.

Wesentlich ist für die neue Barrio-Politik, dass die Armenviertel eindeutig die politische Basis der heutigen Regierung darstellen. Insbesondere in Krisensituationen wie dem Putschversuch 2002 oder im Zusammenhang mit dem Managerstreik und der versuchten Stilllegung der Ölindustrie 2003 konnte sich die Regierung auf die Bevölkerung in den Barrios stützen, die von ihren Nachbarschaften in die Stadt hinunter kam und mit machtvollen Demonstrationen die Kräfteverhältnisse zugunsten der Regierung veränderte. Auch der Versuch, einen Streik der Transportunternehmen zu inszenieren, scheiterte im Februar 2006 letztlich an den neu gegründeten Transportkollektiven aus den Barrios, die umgehend den Transport in Caracas übernahmen.

Zum Zweiten entspricht eine Unterstützung von Basisinitiativen dem ideologischen Kern der bolivarianischen Politikvorstellung. Das Konzept einer die Republik Venezuela konstituierenden vierten Gewalt (Poder Ciudadano) in Gestalt der direkten Demokratie wird nicht nur in ideologischen Programmen und Doktrinen verhandelt, sondern erhielt mit der im Dezember 1999 verabschiedeten Verfassung den Status einer verbindlichen Grundlage staatlichen Handelns. Die Volksmacht wird dabei einerseits als den Staat kontrollierende, andererseits aber diesen durch ihre schöpferische Eigeninitiative erst konstituierende Kraft verstanden. Die Verpflichtung des Staatsapparates ist es daher die Volksmacht zu stärken und Elemente der ,partizipativen und protagonistischen Demokratie“ auszubauen. Entsprechende Passagen lassen sich in der Verfassung zuhauf finden; von besonderer Bedeutung sind hier die Festlegungen, dass die Macht im souveränen Volk wurzele (Art. 5), dass alle Bürger Anrecht auf aktive Partizipation an allen öffentlichen Angelegenheiten haben (Art. 62) und dass „neue Subjekte der Dezentralisierung ... auf der Ebene von Kommunalbezirken, der Zivilgesellschaft, von Wohnvierteln und Nachbarschaftsquartieren" geschaffen werden sollen, an die sukzessive staatliche Kompetenzen in der Ausarbeitung und Durchführung von öffentlichen Investitionsmaßnahmen, Sozialprogrammen und öffentlichen Dienstleistungen zu übertragen sind (Art. 184).

Dieser Linie folgen auch die verschiedenen Misiones, die in vielen Punkten am bestehenden Verwaltungsapparat vorbei mit Unterstützung der Barriobevölkerung implementiert wurden. Die auf Mitarbeit der Nachbarschaft basie- rende Umsetzung von Programmen wie dem Barrio Adentro, die Gesundheitskommitees (siehe Metzger 2007) oder die Runden Tische zur Wasserversorgung zeigten deutlich, dass bislang vom Staat (nicht) erbrachte Dienstleistungen oft besser von den Bewohnern organisiert werden können und dass dies auch zu einer höheren Akzeptanz von politischen Initiativen beiträgt.

Im Ergebnis all dieser Entwicklungen wurde die Einbeziehung der Comunidades zu einem zentralen Pfeiler der bolivarianischen Politik. Gemeinsamer Nenner der verschiedenen Initiativen ist dabei die Idee einer Stärkung der „partizipativen und protagonistischen Demokratie“: Die Bewohner der Armenviertel sollen nicht nur einfach über staatliche Vorhaben informiert werden und dazu Anregungen und oder Bedenken vortragen dürfen -, sondern Investitionspläne und Handlungsprioritäten sollen von den Comunidades erarbeitet und können nur unter Beteiligung der Bewohner umgesetzt werden.

Wie diese Vorstellungen in die Praxis umgesetzt werden, lässt sich gut an zwei Kernbereichen der neuen Barrio-Politik verdeutlichen, mit denen wir uns in Caracas näher auseinandergesetzt haben. Hierbei handelt es sich um die städtische Bodenreform und den Aufbau von kommunalen Planungsräten.

\subsection{Regularisierung der Besitzverhältnisse: \\ Comités de Tierras Urbanas (CTU)}

Die Notwendigkeit einer „städtischen Bodenreform“ ergibt sich direkt aus der Entstehungsgeschichte der Barrios: Da diese als Folge von Besetzungen entstanden, verfugten die Bewohner in der Regel nicht über einen Eigentumstitel für die von ihnen erbauten Häuser. Sie können ihre Häuser deshalb nicht verkaufen, sie können keine Mietverträge abschließen und erhalten keine Kredite für die Instandsetzung oder den Ausbau ihrer Wohnungen. Eine Eigeninitiative der Bewohner zur Verbesserung ihrer Wohnverhältnisse steht damit vor einem entscheidenden Hemmnis, sodass die vorhandene Bereitschaft zur Selbsthilfe nicht genutzt werden kann.

Hinzu kommt auf Seiten des Staates das weitgehende Fehlen von Basisinformationen, die für eine vernünftige Investitionsplanung notwendig wären. Wie viel Bewohner ungefähr in einem Barrio wohnen, wie viele Gebäude es gibt, in welchem Zustand diese sind, wo ein Wasseranschluss vorhanden ist und welche Häuser aufgrund ihrer Lage von Hangrutschungen und Überschwemmungen bedroht sind - all das wissen nur die Bewohner der Nachbarschaften selbst. Die am 4. Februar 2002 mit dem Präsidialdekret 1666 ins Leben gerufene städtische Bodenreform, nach der Bewohner von in Selbsthilfe errichteten und auf besetztem Land stehenden Gebäuden einen Eigentumstitel auf ihre Häuser erhalten können, ist vor diesem Hintergrund ein immenser Schritt, mit dem die Sanierung der Barrios vorangetrieben wird.

Bemerkenswert ist dabei vor allem Umsetzung der Reform, denn die Vergabe 
von Eigentumstiteln erfolgte hier nicht als behördlicher Genehmigungsakt man geht zum Amt, beantragt einen Titel und dieses erteilt bei Vorliegen bestimmter Voraussetzungen die Erlaubnis -, sondern die Ausführung der Bodenreform wurde in die Hände von „Städtischen Bodenkomitees" (Comités de Tierras Urbanas) gelegt. Diese stellen in den Großstädten einen Zusammenschluss von 100 bis 200 Haushalten eines Viertels dar, die ein Landkomitee mit sieben Mitgliedern wählen, das die technischen und behördlichen Schritte bis zur Ausstellung der Bodentitel unterstützt und begleitet.

Flankiert wird der Prozess der Regularisierung durch eine Reihe weiterer Maßnahmen: So werden zur Zeit Flächennutzungspläne festgelegt, die eine ausschließliche Wohnnutzung für die jeweiligen Bereiche definieren und ein Absorbieren der legalisierten Grundstücke durch den konventionellen Immobilienmarkt begrenzen sollen. In einigen Fällen kommt es auch zur Übertragung von kollektiven Eigentumstiteln, so dass die Trägerschaft der Immobilien an die jeweilige Nachbarschaft gebunden bleibt (Antilliano 2005).

Auch der Aufgabenbereich der CTU beschränkt sich bei weitem nicht auf Landvermessung und die Organisation von Besitztiteln. Über diese Bereiche hinaus übernehmen die CTU viele weitere Aufgabenfelder. So gehört es zu den Aufgaben einer CTU, eine „Barrio-Charta“" zu entwerfen. Darin soll die Geschichte des jeweiligen Barrios aufgearbeitet und die Grundregeln der Gemeinschaft festlegt werden. Meist geht damit eine Bestandsaufnahme im Viertel einher, bei der erstmals grundsätzliche Daten erhoben werden. Zusätzlich haben viele CTU angefangen, Unterausschüsse zu bilden, als Verhandlungspartner von Versorgungsunternehmen für Wasser- und Elektrizität, Abwasserund Abfallbeseitigung, die Organisation der kulturellen Aktivitäten, das Management der Sicherheitsinteressen, die Entwicklung von Nachbarschaftsprojekten und andere Ausgaben beschäftigen.

Die CTU werden damit zu einer Art "Scharnier“, über das der Legalisierungsprozess des Grundbesitzes an eine nachbarschaftliche Selbstorganisation gebunden wird. Hinzu kommt die Verkoppelung der Bodenreform mit einer Reihe von finanziellen Unterstützungen, die ebenfalls über die CTU ausgereicht werden und an einen mit der Nachbarschaft erarbeiteten Entwicklungsplan gebunden sind. Entsprechend wurde im Oktober 2005 ein 142,5 Millionen Dollar umfassender Fonds für Barrio-Projekte aufgelegt, dessen Gelder von den CTU's direkt abgerufen werden konnten.

Sowohl die bolivarianischen Medien als auch die der Opposition schätzen die Landreform als eine „stille Revolution“ ein, die nicht nur die Lebensbedingungen der BarriobewohnerInnen deutlich verbessert, sondern auch eine enorme Mobilisierung in den Barrios hervorgerufen hat.

\subsection{Kommunale Räte}

Eine weitere, seit 2006 zunehmend bedeutsame Neuerung der bolivarianischen Kommunalpolitik findet sich in den „Kommunalen Räten“ (Consejos Comunales), die inzwischen weit über die Barrios hinaus an Bedeutung gewonnen haben und eine landesweite Struktur der nachbarschafflichen Selbstverwaltung bilden.

Für die Consejos Comunales gibt es politische und administrative Vorläufer, vor allem in den Consejos Locales de Planificación Publica (CLPP), die vor ein paar Jahren eingeführt wurden, um das in der Verfassung verankerte Beteiligungsversprechen auf lokaler Eben umzusetzen. Sie standen in der Tradition der Bürgerhaushalte von Porto Alegre - scheiterten jedoch weitgehend „an unzureichenden Gesetzen und unwilligen Wahlbeamten auf der mittleren Verwaltungsebene" (Wilpert 2005). Verwiesen wird von Experten auch darauf, dass die CLPP weiterhin auf ein „bürokratisches, repräsentatives und personalisiertes Gemeinwesen" orientierten und damit die Beteiligung der Bewohner auf „Begleitung und Beratung" beschränkte, während Entscheidungen und Genehmigungen weiterhin bei den Verwaltungen verblieben (Interview Borreteran 2006).

Dennoch wurden mit dem Aufbau der CLPP wichtige Grundprinzipien für eine stärkere Beteiligung der Bewohnerschaft an örtlichen Planungsprozessen entwickelt, die in den Strukturen der Kommunalen Räte aufgegriffen und weiterentwickelt wurden. So war bereits im Gesetz für die CLPP festgeschrieben, das jeweils bis zu 20 Prozent des jeweiligen Haushaltes der organisierten Zivilgesellschaft zur Verfügung stehen sollten. Zum Zeitpunkt des Inkrafttretens des Gesetzes waren das 1,2 Billionen Bolivar, umgerechnet rund eine halbe Milliarde Euro, die für Stadtentwicklungsmaßnahmen direkt von den lokalen CLPP abgerufen werden sollten.

Die CLPP waren damit schon allein von der Summe des zur Verfügung gestellten Geldes, das nach geographische Schlüssel an die lokalen Entwicklungsbanken verteilt wurde, eine gewichrige Institution, die neben dem bestehenden Verwaltungssystem eingerichtet wurde und von der Absicht her die Partizipation und den Protagonismus der Bewohner ermöglichen sollte. In der Praxis blockierten die vom alten Verwaltungsapparat durchsetzten Planungsund Genehmigungsprozeduren jedoch den angestrebten basisdemokratischen Planungsprozess. In der Folge verloren die CLPP zunehmend an Bedeutung und wurden durch neue Strukturen ersetzt.

Bereits ab 2004 schlossen sich in einigen Regionen die verschiedenen lokalen Komitees (s.o.) und Misiones zu regehmäßigen Vollversammlungen zusammen, die Unterausschüsse bildeten und dadurch von unten her eine neue Struktur der kommunalen Selbstverwaltung etablierten. Diesen Ansatz der Selbstorganisation begannen die Bundesregierung und die Stadtregierung von Caracas ab 
2005 intensiv zu unterstützen; daraufhin entstanden fast überall in den Barrios von Caracas neue Strukturen der nachbarschaftlichen Interessensvertretung. Nach einem knappen Jahr überwiegend verdeckter Aufbauarbeit wurden schließlich im März 2006 die Consejos Comunales als neue Planungsstruktur vorgestellt und mit eigenen Finanzierungsressourcen ausgestattet. Zeitgleich mit dem ersten öffentlichen Auftritt der Consejo Comunales wurde auch ein neues Gesetz verabschiedet, das deren Befugnisse regelt.

Damit wurde ein zweiter Anlauf unternommen, der die Bürgerbeteiligung auf eine stärker basisdemokratische Grundlage stellte. Ganz offensichtlich schlägt sich das in Abstimmungsmodalitäten und Mehrheitsverhältnissen nieder: Während der 2004 installierte Planungsbeirat (Consejo Metropolitano de Planificacion Publica) noch ein Verhältnis von 45 Regierungsmitarbeitern zu 25 Sprechern von sozialen Organisationen hatte, wurde dieses jetzt zugunsten der Basisorganisationen umgedreht. Per präsidialer Dienstanweisung wurde die neue Zusammensetzung des Planungsbeirats mit einem Verhältnis von 60 Ver treter/innen sozialer Bewegungen und 59 staatlichen Funktionären festgelegt. Damit wurde im Bereich der Stadtplanung den Anforderungen des Planungsgesetzes von 2002 entsprochen und die Gründung von Consejos Comunales forciert.

$\mathrm{Zu}$ den Aufgaben der Consejos Comunales heißt es in einer Informationsschrift des Ministeriums für Soziale Entwicklung und Volkspartizipation: „Der Consejo Comunal ist die am weitesten fortgeschrittene Organisation, die die Nachbarn einer bestimmten Comunidad (einer Nachbarschaft, etwa 200-400 Haushalte, d.A.) bilden können, um die tatsächliche Ausführung der Volksmacht zu übernehmen, d.h. um die von der Comunidad getroffenen Entscheidungen in die Praxis umzusetzen. Er ist die Grundinstanz der Planung, in der das Volk die öffentliche Politik formuliert, ausführt, kontrolliert und bewertet."

Der Consejo Communal kann damit als eine Art Stadtteilregierung begriffen werden, die zusätzlich zur bestehenden Exekutive eingerichtet wird. In der Regel basiert der Consejo personell auf bereits im Viertel vorhandenen Organisationen und Initiativen wie den städtischen Landkomitees, den Gesundheitskomitees, kulturellen Gruppen, Runden Tischen zu Wasser- und Elektrizitätsversorgung, Kooperativen, Volksmilizen, Wahlkampfgruppen etc. Nichtsdestotrotz wird er nicht einfach als Zusammenfassung von Initiativen verstanden, sondern soll ein rechenschaftspflichtiges Ausführungsorgan der Comunidad sein. Die Rückbindung der in den Consejos verankerten Macht an die Bewohner soll durch Bürgerversammlungen sichergestellt werden, deren Entscheidungen für die Consejos bindend sind.

Die Funktionen und Aufgabenbereiche der Consejos werden dabei im Gesetz nicht im Einzelnen trennscharf festgelegt. Konkret nennt das zuständige Ministerium eine ganze Reihe von Zielen:
„1. Die in der Comunidad vorhandenen Basisorganisationen verbinden und die Bildung neuer Organisationen anstoßen, wo es notwendig ist.

2. Einen einheitlichen Arbeitsplan ausarbeiten und seine Ergebnisse auswerten, um die Probleme anzugehen, die die Comunidad mit ihren eigenen Mittel lösen kann.

a) Die materiellen und menschlichen Ressourcen analysieren, die die eigene Comunidad besitzt. b) Die Unterstützung der Nachbarschaft gewinnen, um die Probleme der Comunidad zu lösen.

c) Die Ausarbeitung von Projekten vorantreiben, um die wesentlichen Probleme zu Iösen, die nicht in der Reichweite des Consejo Comunal liegen."

Folgende Punkte betreffen den partizipativen Haushalt oder andere Finanzinstitutionen:

„d) Mit einem Sprecher an der Ausarbeitung des partizipativen Haushaltes teilnehmen.

e) Die Vorschläge der Organisationen der Comunidad zusammenstellen, bearbeiten und Prioritäten setzen.

3. Soziale Kontrolle bei allen Aktivitäten ausüben, die sich in der Comunidad entwickeln, seien sie staatlicher, gemeinschaftlicher oder privater Art (Verpflegung, Bildung, Gesundheit, Kultur, Sport, Infrastruktur, Kooperativen, Missionen, usw.)

4. Die Gründung von Kooperativen und die Ausarbeitung von Projekten endogener Entwicklung vorantreiben.

5. Die Mobilisierung der Comunidad zur Verteidigung ihrer Projekte vorantreiben.

6. Die kollektiven Interessen durch die Ausführung der Aufträge der BürgerInnenversammlung verteidigen.

7. Zur ganzheitlichen (integralen) politischen und kulturellen Entwicklung der Mitglieder der Comunidad beitragen.

8. Die freiwillige soziale Arbeit in jeder einzelnen der Arbeitskommissionen organisieren.

9. Die Ordnung herstellen und gegen die Kriminalität und die Korruption kämpfen. Sprecherinnen und Sprecher benennen, damit der Rat in den höheren Beteiligungsinstanzen präsent ist."

Inhaltlich sind die Consejos Comunales damit als komplette Kommunalverwaltungen ausgestattet. Entscheidend ist jedoch, dass dieses Aufgabenspektrum nich $九$ nur auf dem Papier steht, sondern auch finanziell eine Basis hat.

Die Stadtteilräte können als eine Arbeitsgruppe Bancos Comunales bilden und die Finanzmittel der von ihnen anvisierten Projekte selbst verwalten. Die Summen, um die es sich dabei handelt sind beträchtlich, denn über die Bancos Comunales werden nicht nur die Fördermittel des Fondo Nacional vergeben, in dem verschiedene bereits vorhandene Fördertöpfe zusammengefasst wurden, sondern zusätzlich sollen demnächst die kompletten Steuereinnahmen der neu eingeführten Rohölsteuer für Abbautätigkeiten im Orinocobecken den Consejo Comunales zugute kommen, ebenso wie ein Teil der Erlöse aus den EPS (Empresas de Producción Social). Bereits bei seiner Gründung war der Fondo Nacional de los Consejos Comunales mit 2 Billionen Bolívar (ca. 660 Mill. Euro) ausgestattet (Agencia Bolivariana de Noticias ABN, 09.04.2006). Alleine für die 2500 Wohnungsbauprojekte, die im Laufe des Jahres 2006 von den Consejos Comunales entwickelt worden waren, standen für 2007590 Mrd. Bolivar (ca. 200 Mill. Euro) bereit (Agencia Bolivariana de Noticias ABN, 15.02.2007). Für das kommende Jahr 2008 sind insgesamt 11 Billionen Bolivar (ca. 3,7 Mrd. Euro) für die Komunalen Räte eingeplant (Prensa Presidencial / MinCI, 14.09.2007).

Auf der Grundlage dieser Rahmenbedingungen ist es im letzten Jahr geradezu 
zu einem Boom in der Einrichtung von Consejos Comunales gekommen. Bis August 2006 hatten sich landesweit 14.655 Consejos Comunales gemeldet, von denen die Mehrzahl vor der Verabschiedung des Gesetzes über die Consejos Comunales gegrüindet worden war (ABN, 17.08.06). Im September 2007 waren mehr als 30.000 Consejos Comunales gegründet (Prensa Presidencial / MinCI 14.09.2007). Damit haben sich innerhalb von nur drei Jahren in etwa drei Viertel der venezolanischen Gemeinden entsprechende Selbstverwaltungsorgane gebildet die parallel zum vorhandenen Staatsapparat arbeiten, teilweise dessen Aufgaben übernehmen und direkt von der Bevölkerung kontrolliert werden können.

Obgleich es damit unzweifelhaft wenigstens institutionell zu einer Demokratisierung der Stadtentwicklung kommt, die weit über das, was wir aus europäischen Demokratien kennen, hinausgeht, ist auch dieser Prozess nicht widerspruchsfrei. Auch die neuen Partizipationsstrukturen leiden häufig an der für Venezuela (und weite Teile Lateinamerikas) typischen Kultur von Korruption, Bestechlichkeit und Klientelismus. Immer wieder werden Fälle von Übervorteilung oder unsauberen Abrechnungen - auch und gerade im Umfeld von Wohnungsbauprogrammen, Missiones und Kooperativen - öffentlich. Um dem gegenzusteuern, wurde von der Präsidialkommission die Contraloria Social als verbindlicher Unterausschuss aller Consejos Comunales etabliert. Diese hat das Recht die Akten und die Buchführung der benachbarten Consejos, der staatlichen Verwaltungen, beteiligter Kooperativen und teilweise sogar privaten Unternehmen einzusehen. Damit wurde ein System der horizontalen und vertikalen sozialen Kontrolle eingeführt, das einen partizipativen Ansatz zur Korruptionsbekämpfung verfolgt und eng mit den Alternativ- und Nachbarschaftsmedien verbunden ist.

Probleme, die eher in Zukunft an Bedeutung gewinnen werden, lassen sich in der horizontalen Koordination verschiedener Consejos Comunales und in der Abstimmung zwischen basisdemokratischen Verfahren und hierarchischbürokratischen Entscheidungsmodi in den Verwaltungen finden. Hier ist auf Seiten der Aktivisten bislang die Vorstellung vorherrschend, dass diese Fragen schrittweise in einer Art syndikalistischem Verfahren durch Zusammenschluss mehrerer Kommunalräte zu Bezirksräten, die sich dann wicder zu stadtweiten Räten zusammenschließen, gelöst werden sollten. Entsprechend wurden in jüngster Zeit Consejos Parroquiales (Bezirksräte) konstituiert.

Ungelöst ist in vielen Punkten auch die Frage, welche „öffentliche Gewalt" Kommunaler Rat, Verwaltung oder Präsident - bei Konflikten die Entscheidungshoheit behält. Bislang wird das eher fallweise gelöst, was zu einem unüberschaubaren Frontverlauf zwischen Basismobilisierung und Bürokratie führt. Dass es spätestens mit zunehmender Konsolidierung von Consejos auf übergeordneten Entscheidungsebenen auch dringender wird, Formen der Verschmelzung von administrativ-bürokratischen und basisdemokratischen Entscheidungsmodi zu finden, liegt auf der Hand.
Die derzeitige Regierungspolitik stärkt in dieser Frage die Consejos Comunales. Von zentraler Bedeutung für die zukünftige Stellung der Stadtteilräte dürfte dabei die gegenwärtig diskutierte Verfassungsreform werden, die bislang auf eine weitere Stärkung der Rätestrukturen gegenüber den traditionellen Verwaltungen zielt. In der Neufassung von Artikel 184 der Verfassung soll der Kommunalrat als „Subjekt der Übertragung, der Dekonzentration" definiert werden, „damit der Bundesstaat, die Landes-, Regional- und Lokalregierungen diesen Gebilden der staatsbürgerlichen Partizipation Kompetenzen, Dienstleistungen, Ämter und Mittel übergeben, um die Kommunale Regierung herzustellen." Minister David Velásquez beschrieb die damit einhergehenden Intentionen der venezolanischen Regierung bei der Vorstellung des Entwurfes zur Verfassungsreform wie folgt:

"Unsere Überlegung ist, dass wir auf diese Weise zu kommunalen Regierungen und einem kommunalen Parlamentarismus gelangen, zu einem großsen Gewicht der Gemeinden in der Entscheidungsfindung und in diesen Institutionen, von denen wir meinen, dass ihre Zeit schon abgelaufen ist die sollen." (Presseerkdarung Velasquez, Ministerio del Poder Popular para la Participación y Protección Social, 11.09.2007).

\section{Selbstorganisation und Staat in der Slumentwicklung}

Analysiert man die Entwicklung der Barrios von Caracas, wird schnell deutlich, dass ein Rekurs auf den Staat als maßgebliche Planungs- und Stadtentwicklungsinstanz nicht sehr weit führt. Die Entstehung und Entwicklung informeller Siedlungen ist vielmehr Ergebnis einer "unvollständigen Moderne", in der Stadtentwicklung nicht durch das Zusammenwirken von Märkten und Staatstätigkeit geplant, sondern durch einen Mix aus Marginalisierung der Unterschichten, Klientelsystemen und Selbsthilfe vorangetrieben wurde. Die Mischung aus Staatsversagen, Desinteresse der herrschenden Eliten und aktiver Diskriminierung ist nicht nur Grund für das Entstehen der Barrios, sondern hat jahrzehntelang die bestehenden Formen der Benachteiligung der Barriobewohner zementiert. Schlechte Wohnverhältnisse, soziale und politische Diskriminierung stehen deshalb in Venezuela in einem engen Wechselverhältnis. Ein solcher Zusammenhang ist im globalen Maßstab nicht außergewöhnlich. Auch die vorhandenen Ansätze reguläre Besitzverhältnissen herzustellen und der Versuch, Bewohner stärker in die Gestaltung ihrer Viertel einzubezichen, sind international nicht gerade einzigartig. Was die aktuelle Politik gegenüber informellen Siedlungen in Venezuela aber vom Gros ihrer internationalen Pendants unterscheidet, ist ihre politische Einbindung in das Konzept einer Transformation von Staatlichkeit hin zu einer "partizipativen und protagonistischen Demokratie". Vor diesem Hintergrund findet eine echte Machtverschiebung hin zu den Bewohnerinnen und Bewohnern statt, in der Beteiligung nicht mehr nur eine von oben definierte Spielwiese darstellt, sondern tatsächlich zu einer Veränderung von Kräfteverhältnissen führt. 
Gleichzeitig sind viele Fragen ungeklärt - welchen Weg die weitere Entwicklung der „partizipativen und protagonistischen Demokratie ${ }^{66}$ nimmt, ist noch weitgehend offen. Dabei werden in Zukunft sowohl externe als auch interne Faktoren eine Rolle spielen. Die Finanzierung der neuen Politik ist so nach wie vor von einer hohen Ölrente abhängig (vgl. Lange/Ullrich 2007), so dass ein Absinken des Ölpreises auf dem Weltmarkt voraussichtlich zu erheblichen Schwierigkeiten führen würde. Hinzu kommt die für europäische Beobachter oft nur schwer zu durchschauende Gleichzeitigkeit von De- und Rezentralisierungstendenzen innerhalb des venezolanischen politischen Systems, bei der die Übertragung von Entscheidungsmacht nach unten mit einer Ausweitung von präsidialen Sondervollmachten einhergeht. Obwohl der Präsident diese Vollmachten bisher sehr deutlich für eine Politik zugunsten der Unterklassen genutzt hat, ist hier ein nicht unproblematisches Spannungsverhältnis vorprogrammiert, das in Zukunft an Bedeutung gewinnen könnte.

Ein Unsicherheitsfaktor ist auch die Ungleichzeitigkeit von Organisationsprozessen in verschiedenen Stadtteilen und die Vervielfachung von für ein Problem zuständigen Institutionen. Beide Entwicklungen führen zu einer Vielzahl von "Frontverläufen“, in denen sich nicht nur Basisinitiativen und alte Bürokratien gegenüberstehen, sondern auch politische Instrumentalisierung, lokale und institutionelle Partikularinteressen, Klientel- und Patronagesysteme eine Rolle spielen. Die sich daraus ergebenden Konstellationen sind von außen kaum zu durchschauen und entsprechend schwer ist es, die Zukunft des venezolanischen Experiments vorauszusagen.

Trotz aller Unsicherheit über die weitere Entwicklung bleibt die venezolanische Slumpolitik jedoch eine wichtige Lelktion für den weltweiten Umgang mit informellen Urbanisierungsprozessen. Auf den Punkt gebracht, lautet ihre Kernaussage: eine Integration marginalisierter Wohnsiedlungen kann nur dann erfolgreich sein, wenn sie die Strukturen der Marginalisierung durchbricht und die Bewohner aktiv durch eine Übertragung von Entscheidungsmacht unterstützt.

\section{Literatur}

Antillano, Andrés (2005): La lucha por el reconocimiento y la inclusion en los barrios populares: la experiencia de los Comites de Tierras Urbanas, in: Revista Venezuelana de Economia y Ciencias Sociales, Vol.11, Nr . 3, 205-218.

Aporrea, 15.03.07: Manifiesto del I Encuentro Nacional de Consejos Comunales

Azzellini, Dario (2006): Venezuela Bolivariana. Revolution des 21. Jahrhunderts?, Karlsruhe.

Azzellini, Dario (2007): Von den Mühen der Ebene... Solidarische Ökonomie, kollektive Eigentumsformen, Enteignungen und Arbeitermit- und Arbeiterselbstverwaltung in Venezuela, in: Holm, Andrej u.a. (Hg.): Revolution als Prozess. Selbstorganisierung und Partizipation in Venezuela, Hamburg.

nezuela, Hamburg.
Bolivar, Teolinda (1992): Caracas - Stadt der Barrios, in: ila, Nr. 160, 15-17.

Bolivar, Teolinda u.a. (1994): Problemas de densificación de los barrios caraquenos y sus consecuencias, Research report.
Feireiss, Kristin u.a .(Hg.) (2005): Informal City. Caracas Case, Uster.

Fundación de la Vivienda Popular (FVP) (1991): Tenencia de la tierra en los barrios de Caracas, Caracas.

Gandy, Matthew (2006): Planning, Anti-planning and the infrastructure crisis facing metropolitan Lagos, in: Urban Studies, Vol.43, Nr.2, 371-396.

Grava, Sigurd; Jacob, Klaus; Puszkin-Chevlin, Ana (2001): Disaster Resistant Caracas. Center for Hazards and Risk Research, Earth Institute, Columbia University, Research Report.

Grohmann, Peter (1992): Zwischen Staat, Parteien und eigener Basis, in: ila, Nr. 160, 18-22.

Marcano, E. E. (1993): La Crisis del Agua en Caracas, Caracas.

Holm, Andrej u.a. (Hg.) (2007): Revolution als Prozess: Selbstorganisierung und Partizipation in Venezuela, Hamburg.

Lange, Moritz/ Ullrich, Jan (2007): Soziales Wachstum. Neue Perspektiven in der Wirtschaftspolitik; in Holm, Andrej u.a. (Hg.): Revolution als Prozess. Selbstorganisierung und Partizipation in Venezuela, Hamburg.

Metzger, Wolfram (2007): Der Kampf um Gesundheit für alle; in Holm, Andrej u.a. (Hg.): Revolution als Prozess. Selbstorganisiening und Partizipation in Venezuela, Hamburg.

Neuwirth, Robert (2006): Shadow Cities: A Billion Squatters, A New Urban World, New York.

Perdomo, Rogelio Pérez und Bolívar, Teolinda (1998): Legal Pluralism in Caracas, Venezuela, in: Fernandes, Edésio and Varley, Ann (Hg.): Illegal Cities. Law and urban change in developing countries, London and New York, 123-139.

P.i.s.o. 16 (2004): Welcome to our revolution. Innenansichten des bolivarianischen Prozesses (http://venezuela.open-lab.org/welcome.pdf), München.

Twickel, Christoph (2006): Hugo Chávez. Eine Biographie. Hamburg.

Ullrich, Detlev (1984): Barriopolitik in Caracas (Venezuela), Saarbrücken.

Vallmitjana, Marta u.a. (1991): Plan Rotival. La Caracas que no fue, Caracas.

Wilpert, Gregory (2005): Venezuela's Quiet Housing Revolution: Urban Land Reform [http://trinicenter.com/cgi-bin/selfnews/viewnews.cgi?newsid1126731545,43466,.shtml], 14.09.2005.

UN-Habitat (2003): The challenge of slums. Global report on human settlements, London and Sterling.

Zillmann, Kerstin (1998): Consolidation and densification of informal squatter settlements in Caraca, Venezuela. in: Trialog, No. $57,26-35$.

Davis, Mike (2007): Planet der Slums, Berlin/Hamburg. 